\title{
Prueba de caminata de seis minutos: Recomendaciones y procedimientos
}

\section{Six-Minute Walk Test: Recommendations and procedure}

\author{
Laura Gochicoa-Rangel, * Uri Mora-Romero, ${ }^{*}$ Selene Guerrero-Zúñiga, ${ }^{*}$ Mónica Silva-Cerón, * Silvia Cid-Juárez, ${ }^{*}$ \\ Mónica Velázquez-Uncal,* Adela Durán-Cuéllar,* Isabel Salas-Escamilla, * Roberto Mejía-Alfaro, * Luis Torre-Bouscoulet*
}

*Instituto Nacional de Enfermedades Respiratorias Ismael Cosío Villegas, Ciudad de México.

\begin{abstract}
RESUMEN. La prueba de caminata de 6 minutos (PC6M) evalúa de forma integrada la respuesta de los sistemas respiratorio, cardiovascular, metabóliCo, músculo esquelético y neurosensorial al estrés impuesto por el ejercicio. La integración funcional se analiza mediante la distancia máxima que un individuo puede recorrer durante un período de seis minutos caminando tan rápido como le sea posible. La PC6M constituye una herramienta confiable en el diagnóstico, estadificación, pronóstico y seguimiento de individuos con enfermedades respiratorias crónicas. El presente documento unifica las recomendaciones internaciones del año 2002 y las del 2014 y aporta herramientas necesarias para estandarizar la PC6M.
\end{abstract}

Palabras clave: Caminata de 6 minutos, estandarización, procedimiento, función pulmonar.

\section{INTRODUCCIÓN}

El propósito de la prueba de caminata de seis minutos (PC6M) es medir la distancia máxima que un individuo puede recorrer durante un período de seis minutos caminando tan rápido como le sea posible. ${ }^{1,2}$ De acuerdo con la velocidad a la cual camina una persona, se determinarán los metros recorridos. La PC6M se lleva a cabo en un corredor con longitud de 30 metros, de superficie plana, preferentemente en interiores y evitando el tránsito de personas ajenas a la prueba. ${ }^{1,3}$ La PC6M evalúa, de manera integrada, la respuesta de

\section{Correspondencia:}

Dra. Laura Gochicoa-Rangel

Jefe del Departamento de Fisiología Respiratoria.

Instituto Nacional de Enfermedades Respiratorias Ismael Cosío Villegas.

Calzada de Tlalpan Núm. 4502,

Colonia Sección XVI, 14080, Ciudad de México.

Correo electrónico: drgochis@gmail.com

Trabajo recibido: 15-V-2015; aceptado: 26-V-2015.
ABSTRACT. The six-minute walk test (6MWT) comprehensively evaluates the respiratory, cardiovascular, metabolic, musculoskeletal and sensorineural responses to exercise. Functional integration is analyzed by the maximum distance that an individual is able to walk, as fast as possible, during a period of six minutes. The $6 \mathrm{MWT}$ is a reliable tool for the diagnosis, staging, prognosis and follow-up of patients with chronic respiratory diseases. This document unifies the 2002 and 2014 international recommendations and provides information needed to standardize the 6 MWT.

Keywords: Six-minute walk test, standardization, procedure, lung function.

los sistemas respiratorio, cardiovascular, metabólico, musculoesquelético y neurosensorial que el individuo desarrolla durante el ejercicio. Esta prueba suele considerarse como una prueba submáxima de ejercicio; sin embargo, algunas personas pueden alcanzar su máxima capacidad para ejercitarse..$^{4-8}$

La PC6M ha mostrado ser de utilidad clínica para la clasificación, seguimiento y pronóstico de los pacientes portadores de diversas enfermedades respiratorias. ${ }^{3}$ Además, la prueba permite medir el efecto de intervenciones farmacológicas, quirúrgicas o de rehabilitación sobre la capacidad física de los pacientes. ${ }^{9-11}$ El cambio mínimo clínicamente significativo (CMCS) en sujetos con enfermedades respiratorias crónicas (enfermedad pulmonar obstructiva crónica, hipertensión pulmonar, enfermedades intersticiales) es de 25 a 33 metros con una mediana de 30 metros. $^{3}$

\section{INDICACIONES}

En la Tabla 1 y 2 se muestran las indicaciones y contraindicaciones de la prueba. 
Tabla 1: Indicaciones de la PC6M.

1. Comparaciones pre- y postratamiento en:
a. Trasplante de pulmón
b. Resección de pulmón
c. Cirugía torácica de reducción de volumen
d. Rehabilitación pulmonar
e. EPOC
f. Hipertensión pulmonar
g. Insuficiencia cardíaca

2. Evaluación del estado funcional
a. EPOC
b. Fibrosis quística
c. Insuficiencia cardíaca
d. Enfermedad vascular periférica
e. Fibromialgia
f. Pacientes ancianos

3. Predictor de morbilidad y mortalidad
a. Insuficiencia cardíaca
b. EPOC
c. Hipertensión pulmonar

\section{CONSIDERACIONES TÉCNICAS PARA REALIZAR LA PC6M}

\section{Pasillo o corredor}

1. El pasillo debe estar en interiores, de superficie plana, lo suficientemente ancho para permitir el libre deambular de pacientes que requieren dispositivos de ayuda para la marcha (Figura 1).

2. El pasillo deberá ser exclusivo para la realización de la PC6M. El sujeto que está siendo evaluado es la única persona que puede desplazarse por el pasillo.

3. La longitud del pasillo debe ser de 30 metros (puede realizarse en pasillos de menor longitud; sin embargo, el estándar actual establece que debe ser de 30 metros). ${ }^{1,2}$

4. Debe existir una señal o marca sobre el piso que indique el lugar en el que inicia y termina la distancia de 30 metros. La señal debe ser visible para el técnico que realiza la prueba y para el paciente (Figura 2).

5. Sobre el piso o la pared, deben realizarse marcas visibles cada 3 metros con el fin de que la medición de la distancia recorrida por el paciente sea lo más exacta posible (Figura 3).

6. Deben colocarse dos conos de tráfico: uno a $0.5 \mathrm{~m}$ y otro a 29.5 m de la línea de inicio (Figura 3).

\section{Equipo y material para la PC6M}

1. Escala de Borg impresa en tamaño de letra de 20 puntos, útil en pacientes con limitaciones visuales (Figura 4).
Tabla 2: Contraindicaciones para la PC6M.

\section{Contraindicaciones absolutas}

a. Infarto reciente (3-5 días)

b. Angina inestable

c. Arritmias no controladas que generen síntomas 0 compromiso hemodinámico

d. Síncope

e. Endocarditis, miocarditis o pericarditis aguda

f. Estenosis aórtica grave o sintomática

g. Insuficiencia cardíaca no controlada

h. Tromboembolia pulmonar o infarto pulmonar reciente

i. Trombosis de extremidades inferiores

j. Sospecha de aneurisma disecante

k. Asma no controlada

I. Edema pulmonar

m. Insuficiencia respiratoria aguda

n. Enfermedad no cardiopulmonar aguda que pueda afectar la capacidad de ejercicio o agravarse por ejercicio (infección, tirotoxicosis, insuficiencia renal)

o. Trastorno mental que genere incapacidad para cooperar

Contraindicaciones relativas

a. Estenosis de arteria coronaria izquierda

b. Estenosis valvular moderada

c. Hipertensión arterial en reposo no tratada sistólica $>200 \mathrm{mmHg}$ o diastólica $>120 \mathrm{mmHg}$

d. Taquiarritmias o bradiarritmias

e. Bloqueo AV alto grado

f. Cardiomiopatía hipertrófica

g. Embarazo avanzado o complicado

h. Anormalidades de electrolitos

i. Incapacidad ortopédica para caminar

j. $\mathrm{SpO}_{2}$ en reposo $<85 \%$ (en su caso, se puede realizar con oxígeno suplementario y especificar flujo. Este punto de corte es arbitrario y puede ser modificado de acuerdo a la altitud sobre el nivel del mar)

k. Frecuencia cardíaca en reposo $>120$ latidos por minuto

2. Hoja de recolección de datos (Figura 5).

3. Tabla de trabajo (Figura 6).

4. Cronómetro, estetoscopio y contador de vueltas (Figura 7).

5. Silla (puede ser más de una) o silla de ruedas (Figura 2).

6. Esfigmomanómetro con brazalete que ajuste a las características de la persona que realiza la prueba (Figura 2).

7. Oxímetro de pulso (Figura 8). En las recomendaciones actuales de 2014 se incluye como requisito a la oximetría debido a la importancia de la información clínica que aporta. ${ }^{2}$

8. Desfibrilador automático y carro de paro con nitroglicerina sublingual y salbutamol (inhalador de dosis medida o nebulizador). 


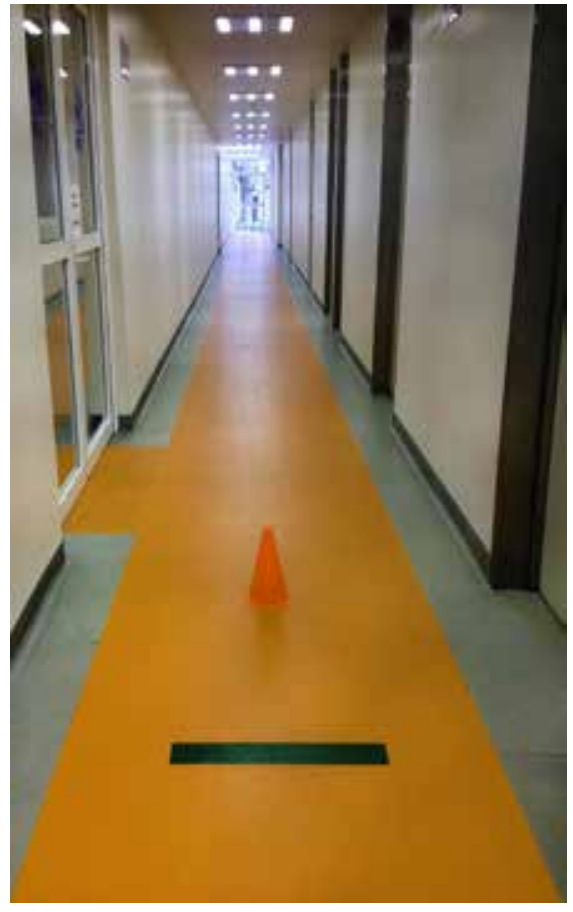

Figura 1:

Pasillo para la PC6M.

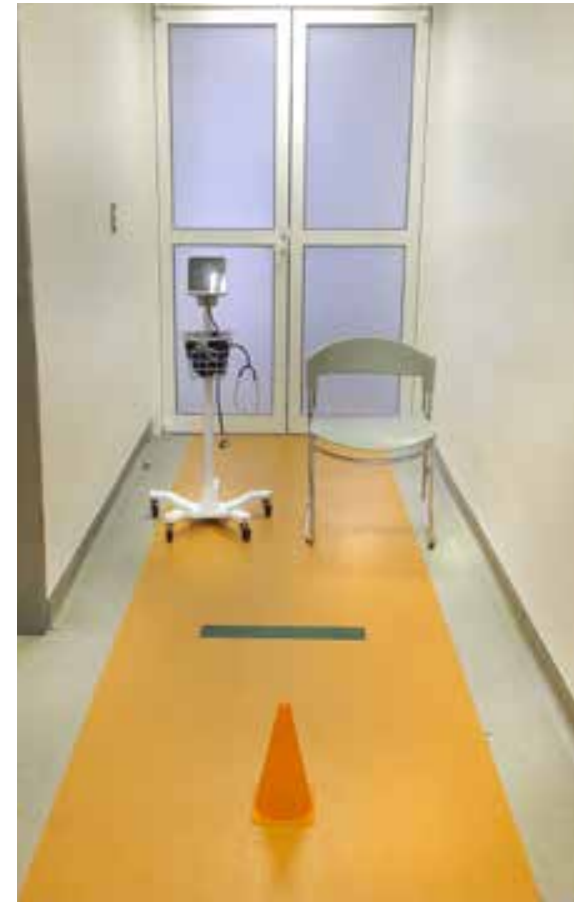

Figura 2: Equipo de la PC6M. Se observa la colocación cerca de la marca de inicio y el cono de tráfico.

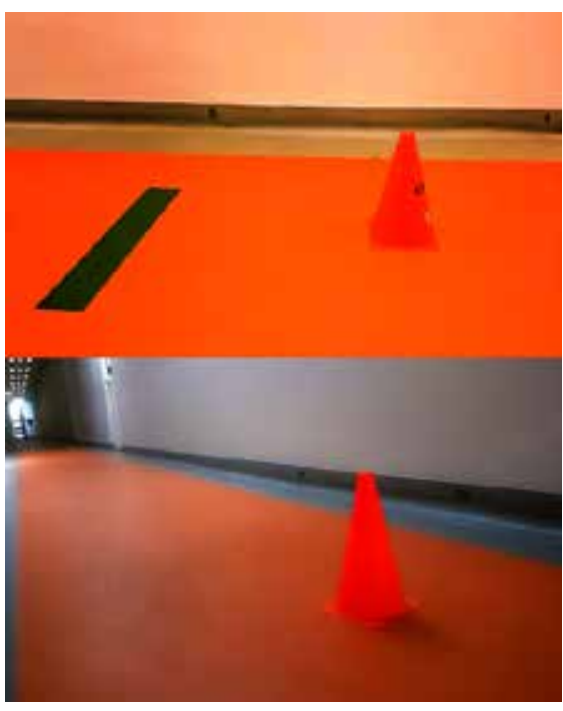

Figura 3: Conos de tráfico y marcas visibles colocadas sobre la pared.
9. Teléfono para casos de emergencia.

10. Estadímetro y báscula.

11. Acceso a una fuente de oxígeno.

12. Plan de emergencia.

\section{PREPARACIÓN DEL PACIENTE ANTES DE LA PC6M}

\section{Al programar la prueba se deben brindar}

\section{las siguientes indicaciones al paciente:}

1. El día de la prueba debe acudir con ropa cómoda y ligera.

2. Zapatos adecuados para realizar ejercicio.

3. Remover el esmalte de uñas, si es el caso.

4. Si el sujeto utiliza dispositivos para asistir a la marcha debe presentarse con ellos el día de la prueba (bastón, prótesis, andadera). Esto se debe anotar en la hoja de recolección de datos para que las pruebas posteriores sean realizadas en las mismas condiciones y puedan ser comparadas.

5. Haber ingerido un alimento ligero.

6. No suspender los medicamentos habituales.

7. No realizar ejercicio vigoroso en las dos horas previas a la prueba.

\begin{tabular}{|c|c|}
\hline \multicolumn{2}{|c|}{ Escala de Borg } \\
\hline Nada & 0 \\
\hline Muy, muy ligera & 0.5 \\
\hline Muy ligera & 1 \\
\hline Ligera & 2 \\
\hline Moderada & 3 \\
\hline Algo intensa & 4 \\
\hline Intensa & 5 \\
\hline \multirow{2}{*}{ Muy intensa } & 6 \\
\hline & 7 \\
\hline \multirow{2}{*}{ Muy, muy intensa } & 8 \\
\hline & 9 \\
\hline Máxima & 10 \\
\hline \multicolumn{2}{|c|}{ Instrucciones para evaluar la Escala de Borg. } \\
\hline \multicolumn{2}{|c|}{$\begin{array}{l}\text { Previo a realizar la prueba deberá mostrar y explicar al paciente la escala } \\
\text { de BORG, haciendo hincapié que deberá reportar un valor (de preferencia } \\
\text { numérico) para "DISNEA» y otro para «FATIGA». }\end{array}$} \\
\hline \multicolumn{2}{|c|}{$\begin{array}{l}\text { Una vez hecha la demostración solicite al paciente que indique el valor en que } \\
\text { se encuentra en este momento, con la siguiente frase: }\end{array}$} \\
\hline \multicolumn{2}{|c|}{$\begin{array}{l}\text { ¿Señale usted en este momento" } \\
\text { ¿Cuánto le falta el aire? } \\
\text { ¿El grado de cansancio en las piernas? }\end{array}$} \\
\hline
\end{tabular}

Figura 4: Escala de Borg para disnea y fatiga. 


\section{Hoja de trabajo}

\section{Prueba de caminata de 6 minutos}

Nombre:

Apellido materno

No. Expediente:

(AAAA/MM/DD)
Fecha:

(AAAA/MM/DD)
Nombre (s)

Peso:

Diagnóstico:

Edad:

:

FC Máx:

Talla: (cm) Género:

Técnico:

Prueba «A»

\begin{tabular}{|c|l|l|l|l|l|}
\cline { 2 - 6 } \multicolumn{1}{c|}{} & FC & $\mathrm{SpO}_{2}$ & $\begin{array}{c}\text { BORG } \\
\text { Disnea }\end{array}$ & $\begin{array}{c}\text { BORG } \\
\text { Fatiga }\end{array}$ & TA \\
\hline Reposo & & & & & \\
\hline Vuelta 1 & & & & & \\
\hline Vuelta 2 & & & & & \\
\hline Vuelta 3 & & & & & \\
\hline Vuelta 4 & & & & & \\
\hline Vuelta 5 & & & & & \\
\hline Vuelta 6 & & & & & \\
\hline Vuelta 7 & & & & & \\
\hline Vuelta 8 & & & & & \\
\hline Vuelta 9 & & & & & \\
\hline Vuelta 10 & & & & & \\
\hline Vuelta 11 & & & & & \\
\hline Vuelta 12 & & & & & \\
\hline
\end{tabular}

\begin{tabular}{|c|l|l|l|l|l|}
\hline Final & & & & & \\
\hline Minuto 1 & & & & & \\
\hline Minuto 3 & & & & & \\
\hline Minuto 5 & & & & & \\
\hline
\end{tabular}

Distancia

Se detuvo: Sí

Motivo

Mareo:

metros

No

Dolor:

Disnea:

Angina:

Otros:

Prueba «B»

\begin{tabular}{|c|l|l|l|l|l|}
\cline { 2 - 6 } \multicolumn{1}{c|}{} & FC & $\mathrm{SpO}_{2}$ & $\begin{array}{c}\text { BORG } \\
\text { Disnea }\end{array}$ & $\begin{array}{c}\text { BORG } \\
\text { Fatiga }\end{array}$ & TA \\
\hline Reposo & & & & & \\
\hline Vuelta 1 & & & & & \\
\hline Vuelta 2 & & & & & \\
\hline Vuelta 3 & & & & & \\
\hline Vuelta 4 & & & & & \\
\hline Vuelta 5 & & & & & \\
\hline Vuelta 6 & & & & & \\
\hline Vuelta 7 & & & & & \\
\hline Vuelta 8 & & & & & \\
\hline Vuelta 9 & & & & & \\
\hline Vuelta 10 & & & & & \\
\hline Vuelta 11 & & & & & \\
\hline Vuelta 12 & & & & & \\
\hline
\end{tabular}

\begin{tabular}{|c|l|l|l|l|l|}
\hline Final & & & & & \\
\hline Minuto 1 & & & & & \\
\hline Minuto 3 & & & & & \\
\hline Minuto 5 & & & & & \\
\hline
\end{tabular}

Distancia

Se detuvo:

Motivo
Sí

Mareo:

Dolor: metros

No

Comentarios:

Figura 5: Hoja de recolección de datos para la PC6M. 


\section{El día de la prueba y a la llegada del paciente se deberá:}

1. Verificar la solicitud de estudio debidamente requisitada, con número de folio, fecha y hora correctas.

2. Recibir y presentarse con el paciente; confirmar que sus datos sean correctos (nombre y fecha de nacimiento).

3. En caso de que el paciente hable algún dialecto, deberá acompañarlo un intérprete para explicarle el procedimiento.

4. Explicar al paciente el objetivo de la prueba. La frase más sencilla recomendada es la siguiente: «La caminata de 6 minutos es una prueba que consiste en caminar lo más rápido que le sea posible durante un período de 6 minutos en un pasillo plano.»

5. Verificar que no existan contraindicaciones para realizar la prueba (Tabla 2).

6. Informar al personal médico del laboratorio si el paciente presenta una o más contraindicaciones para que se realice la prueba.

7. En caso de que el paciente utilice oxígeno suplementario de manera ambulatoria, la prueba debe realizarse con el aporte de oxígeno habitual, con el flujo constante. Si el propósito es comparar la distancia recorrida

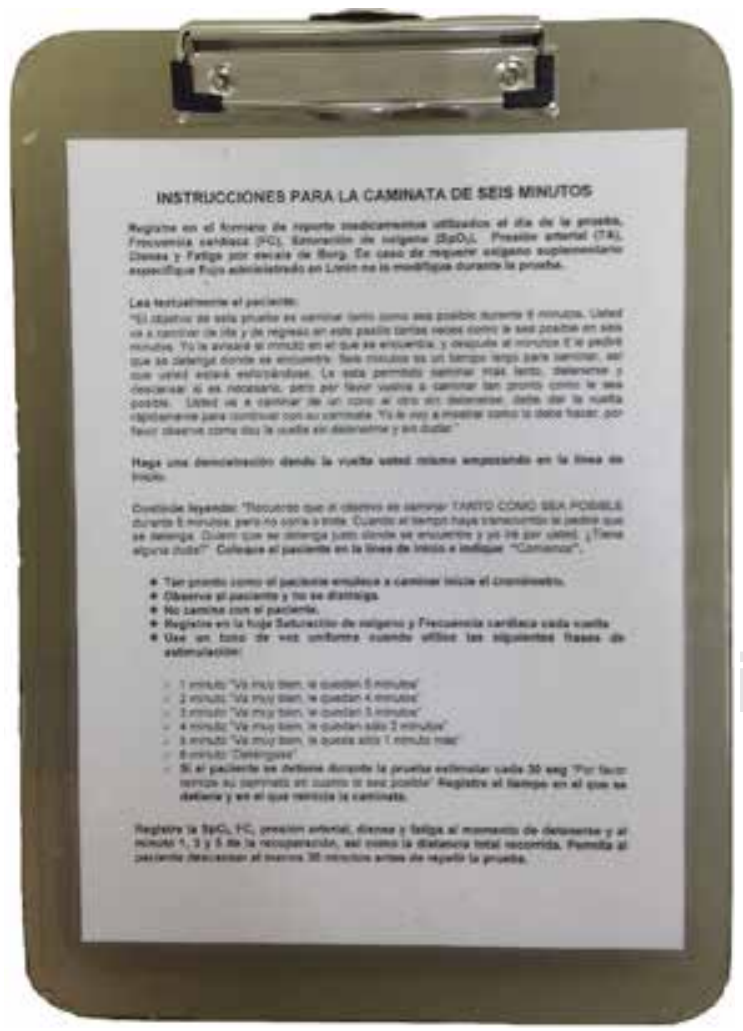

Figura 6: Tabla de trabajo. Se observan las instrucciones estandarizadas de la PC6M. entre dos pruebas, se debe utilizar el mismo dispositivo para la administración de oxígeno y el mismo flujo. En cualquiera de los casos debe consignarse en la hoja de recolección de datos.

8. En caso de que se requiera incrementar el flujo de oxígeno durante la prueba deberá registrarse en la hoja de trabajo (ver la sección de recomendaciones de uso de oxígeno).

9. Informar el tipo de dispositivo de administración de oxígeno suplementario; por ejemplo, oxígeno líquido concentrado fijo o portátil, y si el flujo es continuo o a demanda.

\section{Procedimiento de la PC6M}

1. Medir y pesar al paciente de forma estandarizada y registrarlo en la hoja de trabajo.

2. Calcular y registrar la frecuencia cardíaca máxima esperada con la fórmula (220-edad del paciente).

3. Medir la presión arterial y registrar los valores basales.

4. Solicitar al paciente que permanezca en posición sedente al menos 15 minutos antes de la prueba. ${ }^{2}$
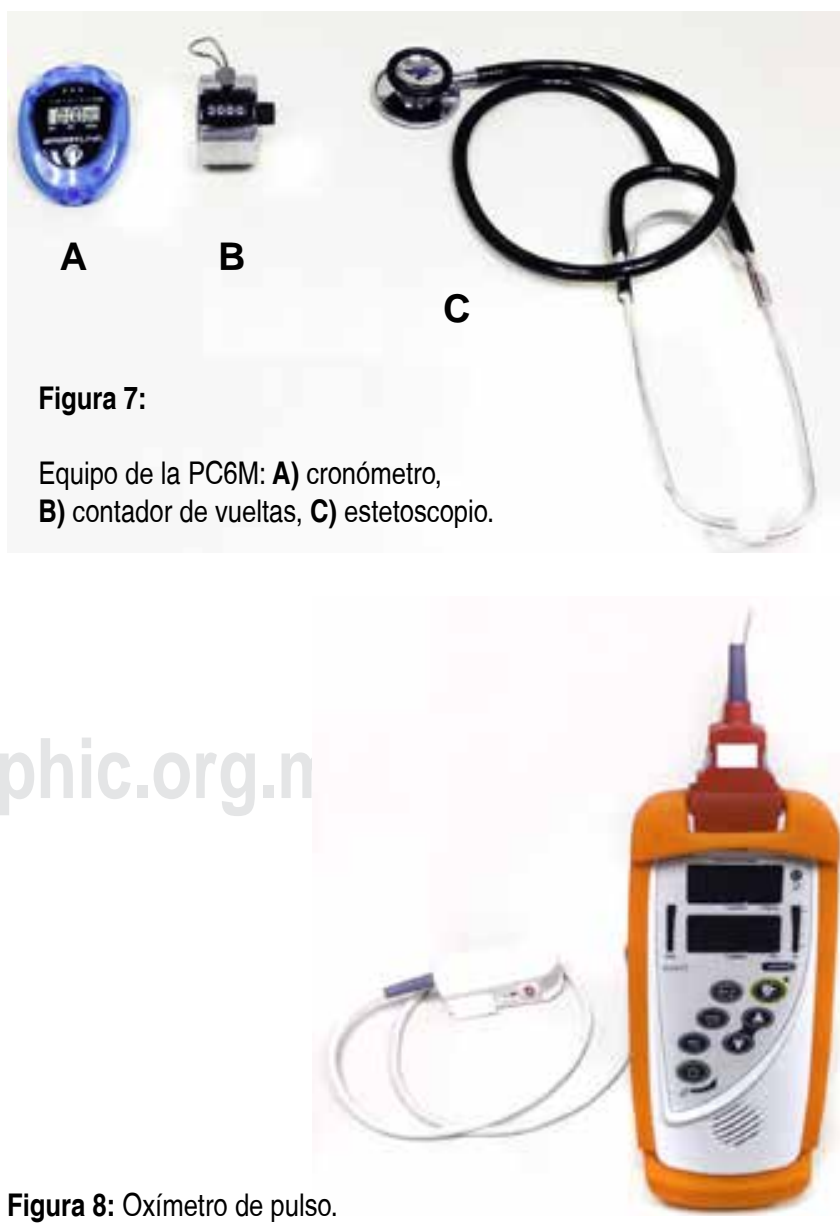

Figura 8: Oxímetro de pulso. 
5. Colocar el oxímetro de pulso y registrar la $\mathrm{SpO}_{2}$ y la frecuencia cardíaca en reposo y registrar los valores basales

6. Verificar que el contador de vueltas se encuentre en cero y el cronómetro programado para seis minutos.

7. Explicar al paciente en qué consiste la Escala de Borg y registrar el valor basal (Figura 4).

8. Leer textualmente las instrucciones al paciente (no agregar oraciones o eliminar palabras); mostrar por escrito a aquellos pacientes con audición disminuida:

9. Leer textualmente al paciente: «El objetivo de esta prueba es caminar tanto como sea posible durante 6 minutos. Usted va a caminar de ida y de regreso en este pasillo tantas veces como le sea posible en seis minutos. Yo le avisaré el paso de cada minuto y después, al minuto 6 , le pediré que se detenga donde se encuentre. Seis minutos es un tiempo largo para caminar, así que usted estará esforzándose. Le está permitido caminar más lento, detenerse y descansar si es necesario, pero por favor vuelva a caminar tan pronto como le sea posible. Usted va a caminar de un cono al otro sin detenerse, debe dar la vuelta rápidamente para continuar con su caminata. Yo le voy a mostrar cómo lo debe hacer, por favor observe cómo doy la vuelta sin detenerme y sin dudar.»

10. Hacer una demostración dando la vuelta usted mismo empezando en la línea de inicio.

11. Continuar leyendo: «Recuerde que el objetivo es caminar tanto como sea posible durante 6 minutos, pero no corra o trote. Cuando el tiempo haya transcurrido le pediré que se detenga. Quiero que se detenga justo donde se encuentre y yo iré por usted. ¿Tiene alguna duda?»

\section{Inicio de la prueba}

1. Colocar al paciente en la línea de inicio e indicar «Comience».

2. Iniciar el cronómetro tan pronto como el paciente empiece a caminar.

3. Observar al paciente atentamente.

4. No caminar con el paciente o atrás de él.

5. Registrar en la hoja saturación de oxígeno y frecuencia cardíaca cada vuelta.

6. Usar un tono de voz uniforme cuando diga las siguientes frases de estimulación:

a. Después de 1 minuto diga al paciente: «Va muy bien, le quedan 5 minutos.»

b. Al completar el minuto 2 diga: «Va muy bien, le quedan 4 minutos.»

c. Al minuto 3 diga al paciente: «Va muy bien, le quedan 3 minutos.» d. Al minuto 4 diga al paciente: «Va muy bien, le quedan sólo 2 minutos.»

e. Al minuto 5 diga al paciente: «Va muy bien, le queda sólo 1 minuto más.»

f. Cuando complete 6 minutos diga al paciente: «Deténgase donde está.»

7. Si el paciente se detiene durante la prueba estimular cada 30 segundos diciéndole: «Por favor reinicie su caminata en cuanto le sea posible.» Registrar el tiempo en el que se detiene y en el que reinicia la caminata. Si el paciente se niega a continuar o usted considera que ya no debe seguir realizando la prueba, acercar una silla y anotar las razones para detener la caminata.

8. Las siguientes son indicaciones para interrumpir inmediatamente la prueba. En tal caso, se debe acercar una silla y anotar en la hoja de trabajo los metros caminados, el minuto en que se detuvo y las razones para detenerla. Avisar inmediatamente al personal médico del laboratorio:

a. dolor torácico;

b. disnea intolerable;

c. marcha titubeante;

d. sudoración, palidez;

e. calambres en miembros pélvicos;

f. palidez o apariencia de desvanecimiento inminente;

g. que el paciente lo solicite;

h. oximetría de pulso $<80 \%$. Este punto de corte se propone por razones de seguridad de la PC6M; ;,12 se ha reportado una incidencia muy baja de eventos adversos graves; ${ }^{13}$

a. Si durante la realización de la prueba el paciente presenta una $\mathrm{SpO}_{2}<80 \%$ se le solicitará que se detenga, en el caso de incrementar la $\mathrm{SpO}_{2}$ $>80 \%$ se le solicitará reinicie la caminata hasta que complete los 6 minutos. ${ }^{2,13}$

9. Al completar 6 minutos y el paciente se haya detenido, se debe acercar una silla e indicarle que se siente; se debe registrar cuanto antes la saturación de oxígeno, frecuencia cardíaca, presión arterial, disnea y fatiga (Escala de Borg). Estos parámetros se deben registrar también después de uno, tres y cinco minutos de haber concluido la caminata.

10. Marcar el punto donde el paciente se detuvo.

11. Registrar el número de vueltas marcadas en el contador así como los metros recorridos al final (en la última vuelta parcial).

12. Calcular la distancia total caminada.

13. Anotar los metros caminados, redondeando al metro más cercano.

14. Felicitar al paciente por el esfuerzo realizado. 
15. Calcular el porcentaje alcanzado de la frecuencia cardíaca máxima para el paciente.

16. Colocar al paciente en posición sedente 30 minutos y después repetir la prueba con la misma metodología. Es recomendable que la prueba se realice por duplicado con 30 minutos de diferencia. Queda a criterio del director médico del laboratorio si en algunos casos sólo se realiza en una ocasión.

17. Generar el reporte de los resultados

\section{Informe de los resultados}

En el informe final de la PC6M se deben incluir:

1. Datos del paciente: nombre, edad, peso y estatura.

2. Nombre completo del técnico que realizó la prueba.

3. Diagnóstico o indicación de la prueba.

4. Deben incluirse los resultados de las variables medidas antes, durante y después de la PC6M (signos vitales, Escala de Borg, saturación de oxígeno).

5. En caso de que el paciente haya presentado síntomas que obligaron a interrumpir la prueba se debe mencionar en el informe.

6. El número de metros caminados. Si se realizan dos pruebas se suele reportar la prueba con la mayor cantidad de metros recorridos.

7. Opcionalmente se puede consignar:

a. Porcentaje (\%) del predicho de los metros caminados, y en ese caso especificar la ecuación de referencia utilizada. En las Tablas 3 y 4 se muestran algunas ecuaciones de referencia para la PC6M en adultos y niños.

b. Nadir de $\mathrm{SpO}_{2}$ durante la prueba. Se ha propuesto recientemente como un indicador de gravedad y como índice para predecir mortalidad en pacientes con enfermedades intersticiales. Este nadir de $\mathrm{SpO}_{2}$ a menudo no ocurre al final de la PC6M.

\section{Recomendaciones generales}

El personal técnico debe estar capacitado sobre la forma estandarizada de realizar la antropometría y sobre la prueba. Debe contar con el entrenamiento en soporte vital básico $y$, de ser posible, en soporte vital avanzado.

La PC6M puede ser aplicada por un solo técnico; sin embargo, nuestra recomendación es que sean dos, debido a que se minimizan los errores en la ejecución y aumenta la seguridad del paciente durante la prueba.

\section{RECOMENDACIONES PARA EL USO DE OXÍGENO}

1. Se utilizará oxígeno suplementario en el caso de que la $\mathrm{SpO}_{2}$ basal sea $<90 \%$ y el paciente previamente no utilizará $\mathrm{O}_{2}$ suplementario (este punto de corte de $\mathrm{SpO}_{2}$ podrá ajustarse de acuerdo a la altitud).

2. Se ajustará el flujo de $\mathrm{O}_{2}$ en pacientes con indicación ambulatoria de $\mathrm{O}_{2}$ suplementario y que el día de la prueba presenten una $\mathrm{SpO}_{2}<90 \%$ con el flujo prescrito.

3. Si durante la prueba basal el paciente presenta una $\mathrm{SpO}_{2}<90 \%$ o se requiere incrementar el flujo de $\mathrm{O}_{2}$

Tabla 3: Ecuaciones de predicción de la prueba de caminata de 6 minutos en adultos.

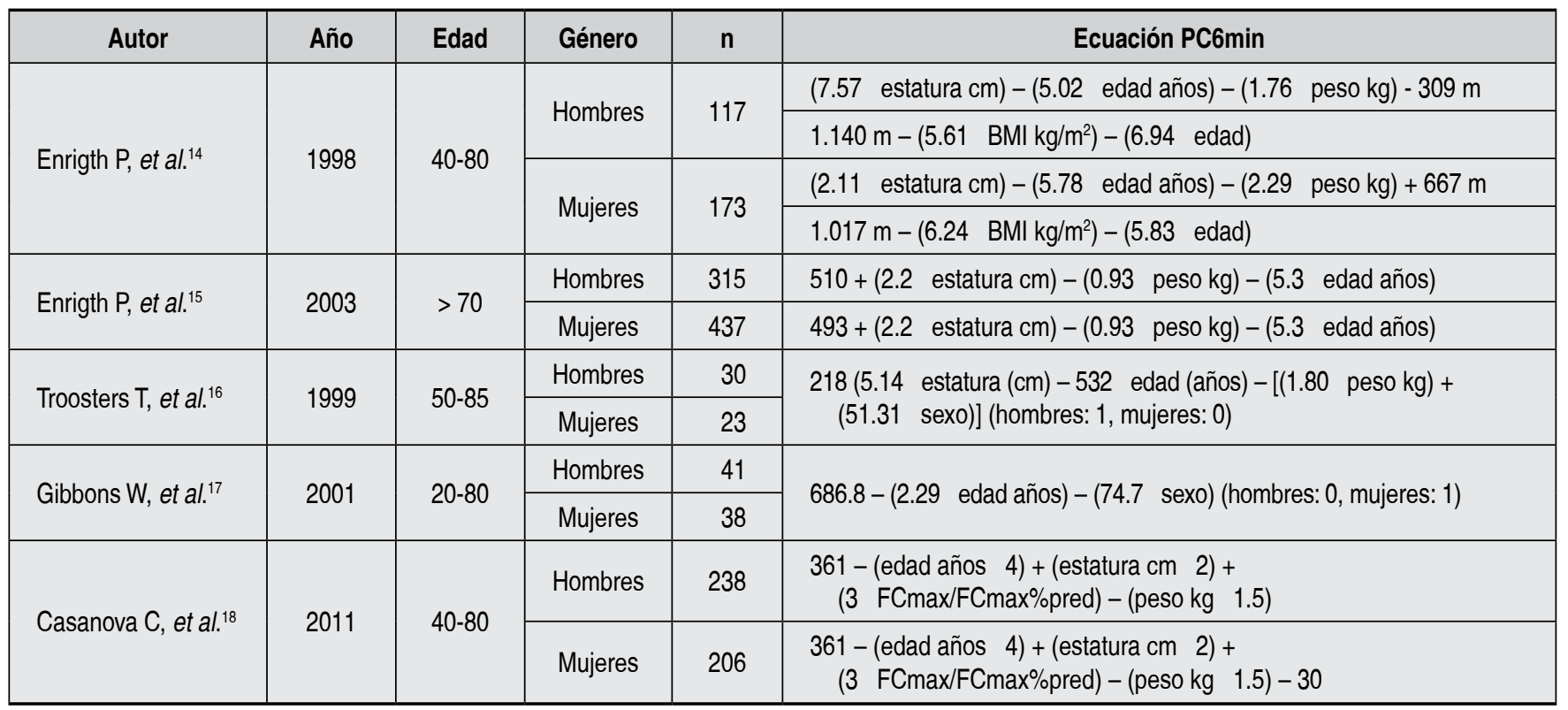


Neumol Cir Torax. 2019; 78 (Supl 2): S164-S172

Tabla 4: Ecuaciones de predicción de la prueba de caminata de 6 minutos en niños.

\begin{tabular}{|c|c|c|c|c|c|}
\hline Autor & Año & Edad & Género & $\mathbf{n}$ & Ecuación PC6min \\
\hline Li AM, et al. ${ }^{19}$ & 2007 & $7-16$ & Mujeres & 640 & $526.79+($ dif FC 1.66) + [estatura $(\mathrm{cm})$ 0.62] \\
\hline Geiger $\mathrm{R}$, et al. ${ }^{20}$ & 2007 & $3-18$ & Hombres & 280 & $\begin{array}{l}196.72+(39.81 \text { edad años })-(1.36 \text { edad } 2 \text { años })+ \\
(132.28 \text { x estatura metros })\end{array}$ \\
\hline \multirow{2}{*}{ Priesnitz CV, et al..$^{21}$} & \multirow{2}{*}{2009} & \multirow{2}{*}{$6-12$} & Hombres & 92 & \multirow{2}{*}{$\begin{array}{l}145.343+(11.78 \text { edad años })+(292.22 \text { estatura } m)+ \\
(0.611 \text { dif FC })-(2.684 \text { peso } \mathrm{kg})\end{array}$} \\
\hline & & & Mujeres & 96 & \\
\hline Gatica D, et al. ${ }^{22}$ & 2012 & $6-14$ & Hombres & 92 & $\begin{array}{l}331,404+(158,523 \text { estatura } \mathrm{m})+(11,945 \text { edad años })- \\
(2,139 \text { peso } \mathrm{kg})+(70,221 \mathrm{FC} \text { de reserva })\end{array}$ \\
\hline Ulrich S, et al. ${ }^{23}$ & 2013 & $5-17$ & Mujeres & 252 & 391.9 estatura $(\mathrm{m})-2.41$ peso $(\mathrm{kg})+140.2$ \\
\hline Goemans $\mathrm{N},{ }^{24}$ & 2013 & $5-12$ & Hombres & 352 & $\begin{array}{l}86.795+74.547 \text { edad (años) }-3.018 \text { edad }^{2} \text { (años) + } \\
\quad 63.204 \text { estatura }(m)\end{array}$ \\
\hline
\end{tabular}

se repetirá la prueba con la nueva titulación de $\mathrm{O}_{2}$ suplementario y se reportará la PC6M con mayor número de metros recorridos.

4. Se considera como desaturación significativa para modificar el aporte de $\mathrm{O}_{2}$ suplementario durante la PC6M:

a. Disminución $4 \%$ de la $\mathrm{SpO}_{2}$ basal y la $\mathrm{SpO}_{2}$ sea $<$ $85 \%$ en adultos.

b. Disminución $4 \%$ de la $\mathrm{SpO}_{2}$ basal y la $\mathrm{SpO}_{2}$ sea $<$ $90 \%$ en niños.

Cualquier modificación en la utilización de $\mathrm{O}_{2}$ suplementario deberá ser anotado en la hoja de trabajo.

\section{CONSIDERACIONES PARA LA PC6M EN NIÑOS}

La PC6M se ha utilizado en la evaluación de la capacidad física en niños sanos ${ }^{25}$ y con enfermedades respiratorias crónicas como fibrosis quística, ${ }^{26}$ bronquiolitis obliterante y cardiopatías. ${ }^{27}$ Existen varias ecuaciones de referencias de la PC6M en niños y adolescentes que siguen las recomendaciones de ATS 2002; sin embargo, distintos grupos de trabajo al interrogar la percepción del esfuerzo físico realizado durante la prueba (sobre todo en menores de 11 años), se enfrentan a una limitada asociación o inconsistencia en la asignación de un «número» o una «palabra» para «disnea» o «fatiga». Se ha utilizado la Escala de Borg, otras iniciativas proponen una modificación de la Escala de Borg ${ }^{28}$ sustituyendo la frase que evalúa el grado de disnea y fatiga por una escala visual. Otras escalas que han demostrado ser útiles en la percepción de esfuerzo físico en niños es la escala OMNI. ${ }^{29}$

\section{RECOMENDACIONES PARA INVESTIGACIÓN EN PC6M}

En las más recientes revisiones de la PC6M se han puesto de manifiesto algunos puntos de interés que se abordan en el desarrollo de este documento, como el reporte del nadir de $\mathrm{SpO}_{2}$, que nos aportará información valiosa sobre la gravedad y pronóstico.

Se deberá conocer la seguridad para realizar la PC6M, sobre todo en pacientes con enfermedades respiratorias de grado modero o grave y que son aquellos que durante la prueba registran un nivel de $\mathrm{SpO}_{2}<80 \%$.

Es de gran importancia realizar la validación de las ecuaciones de referencia disponibles en niños o en su defecto realizar las ecuaciones en población mexicana; además, se requiere validar las escalas de síntomas en la población pediátrica.

\section{REFERENCIAS}

1. ATS Committee on Proficiency Standards for Clinical Pulmonary Function Laboratories. ATS statement: guidelines for the six-minute walk test. Am J Respir Crit Care Med 2002;166(1):111-117. 
2. Holland AE, Spruit MA, Troosters T, et al. An official European Respiratory Society/American Thoracic Society technical standard: field walking tests in chronic respiratory disease. Eur Respir J 2014;44(6):1428-1446. doi: 10.1183/09031936.00150314.

3. Singh SJ, Puhan MA, Andrianopoulos V, et al. An official systematic review of the European Respiratory Society/American Thoracic Society: measurement properties of field walking tests in chronic respiratory disease. Eur Respir J 2014;44(6):1447-1478. doi: 10.1183/09031936.00150414.

4. Watz H, Pitta F, Rochester C, et al. An official European Respiratory Society statement on physical activity in COPD. Eur Respir J 2014;44(6):1521-1537. doi: 10.1183/09031936.00046814

5. Luxton N, Alison JA, Wu J, Mackey MG. Relationship between field walking tests and incremental cycle ergometry in COPD. Respirology 2008;13(6):856-862. doi: 10.1111/j.1440-1843.2008.01355.x.

6. Holland A, Knapman L, Brazzale DJ, et al. The 6-minute walk test elicits high but submaximal cardiorespiratory responses in interstitial lung disease. Am J Respir Crit Care Med 2010;181:A2968.

7. Deboeck G, Niset G, Vachiery JL, Moraine JJ, Naeije R. Physiological response to the six-minute walk test in pulmonary arterial hypertension. Eur Respir J 2005;26(4):667-672.

8. Blanco I, Villaquirán C, Valera JL, et al. Peak oxygen uptake during the six-minute walk test in diffuse interstitial lung disease and pulmonary hypertension. Arch Bronconeumol 2010;46(3):122-128. doi: 10.1016/j. arbres.2009.12.005.

9. Lacasse Y, Goldstein R, Lasserson TJ, Martin S. Pulmonary rehabilitation for chronic obstructive pulmonary disease. Cochrane Database Syst Rev 2006;(4): CD003793.

10. Puhan M, Scharplatz M, Troosters T, Walters EH, Steurer J. Pulmonary rehabilitation following exacerbations of chronic obstructive pulmonary disease. Cochrane Database Syst Rev 2009;(1):CD005305. doi: 10.1002/14651858.CD005305.

11. Holland A, Hill C. Physical training for interstitial lung disease. Cochrane Database Syst Rev 2008;(4):CD006322. doi: 10.1002/14651858. CD006322.

12. American Thoracic Society/American College of Chest Physicians. ATS/ACCP Statement on cardiopulmonary exercise testing. Am J Respir Crit Care Med 2003;167(2):211-277.

13. Jenkins S, e ins N. Six-minute walk test: observed adverse events and oxygen desaturation in a large cohort of patients with chronic lung disease. Intern Med J 2011;41(5):416-422. doi: 10.1111/j.14455994.2010.02169.x.

14. Enright PL, Sherrill DL. Reference equations for the six-minute walk in healthy adults. Am J Respir Crit Care Med 1998;158(5 Pt 1):1384-1387.

15. Enright PL, McBurnie MA, Bittner V, et al; Cardiovascular Health Study. The 6-min walk test: a quick measure of functional status in elderly adults. Chest 2003;123(2):387-398.
16. Troosters T, Gosselink R, Decramer M. Six minute walking distance in healthy elderly subjects. Eur Respir J 1999;14(2):270-274.

17. Gibbons WJ, Fruchter N, Sloan S, Levy RD. Reference values for a multiple repetition 6-minute walk test in healthy adults older than 20 years. J Cardiopulm Rehabil 2001;21(2):87-93.

18. Casanova C, Celli BR, Barria P, et al.; Six Minute Walk Distance Project (ALAT). The 6-min walk distance in healthy subjects: reference standards from seven countries. Eur Respir J 2011;37(1):150-156. doi: 10.1183/09031936.00194909.

19. Li AM, Yin J, Au JT, et al. Standard reference for the six-minute-walk test in healthy children aged 7 to 16 years. Am J Respir Crit Care Med 2007;176(2): 174-180.

20. Geiger R, Strasak A, Treml B, et al. Six-minute walk test in children and adolescents. J Pediatr 2007;150(4):395-399.

21. Priesnitz CV, Rodrigues GH, Stumpf Cda S, et al. Reference values for the 6-min walk test in healthy children aged 6-12 years. Pediatr Pulmonol 2009; 44(12):1174-1179. doi: 10.1002/ppul.21062.

22. Gatica D, Puppo H, Villarroel $\mathrm{G}$, et al. Reference values for the 6-minutes walking test in healthy Chilean children. Rev Med Chil 2012;140(8):1014-1021. doi: 10.4067/S0034-98872012000800007.

23. Ulrich $\mathrm{S}$, Hildenbrand $\mathrm{F}$, Treder $\mathrm{U}$, et al. Reference values for the 6-minute walk test in healthy children and adolescents in Switzerland. BMC Pulm Med 2013;13:49. doi: 10.1186/1471-2466-13-49.

24. Goemans N, Klingels K, van den Hauwe M, et al. Six-minute walk test: reference values and prediction equation in healthy boys aged 5 to 12 years. PLoS One 2013;8(12):e84120. doi: 10.1371/journal. pone. 0084120

25. Lesser DJ, Fleming MM, Maher CA, Kim SB, Woo MS, Keens TG. Does the 6-min walk test correlate with the exercise stress test in children? Pediatr Pulmonol 2010;45 (2):135-140. doi: 10.1002/ppul.21125.

26. Cunha MT, Rozov T, de Oliveira RC, Jardim JR. Six-minute walk test in children and adolescents with cystic fibrosis. Pediatr Pulmonol 2006;41(7):618-622.

27. Moalla W, Gauthier R, Maingourd Y, Ahmaidi S. Six-minute walking test to assess exercise tolerance and cardiorespiratory responses during training program in children with congenital heart disease. Int J Sports Med 2005;26(9): 756-762.

28. Zenteno D, Puppo H, González R, Kogan R. Test de marcha de 6 minutos en pediatría. Neumol Pediatr 2007;2(2):109-114.

29. Robertson RJ, Goss FL, Boer NF, et al. Children's OMNI scale of perceived exertion: mixed gender and race validation. Med Sci Sports Exerc 2000;32(2):452-458.

Los autores declaran no tener conflicto de intereses. 